\title{
Análisis de la eficiencia de un motor diésel, mediante el estudio estadístico de fallos de filtros de combustible.
}

Analysis of the efficiency of a diesel engine, through the statistical study of fuel filter failures.

Alejandro Huilca Álvarez. ${ }^{1} \&$ Silvana Haro Acosta. ${ }^{2}$

\begin{abstract}
DOI: https://doi.org/10.33262/cienciadigital.v3i3.660

In the automotive industry a variety of diesel fuel filters are used, in order to provide a better filtering capacity. The purpose of the study is to determine the types of filter used in the fuel supply system in the same sense, in the performance and efficiency of a diesel engine, in the statistical mathematical analysis of the data on the faults that have occurred. Analyzed vehicles that use the 3 recurring filter brands in the city of Riobamba province of Chimborazo, stating a hypothesis that affirms or denies, the positive change in engine efficiency, taking into account a sample of the city's automotive fleet. The results were the type of filter used not only in the efficiency of the engine, but other operational components may influence what is in the present, for the reason that this study is only a probabilistic premise through the result of the statistical mathematical analysis, being able to serve in the future for an exhaustive investigation of the conditions and parameters that influence the efficiency of an engine.
\end{abstract}

Keywords Efficiency, diesel engine, fuel filtering, statistical mathematical analysis; Impurities

\section{Resumen:}

En la industria automotriz se emplea una diversidad de filtros para combustible diésel, con la finalidad de proporcionar una mejor capacidad de filtrado. El objeto

\footnotetext{
${ }^{1}$ Escuela Superior Politécnica de Chimborazo, Facultad de Mecánica, Carrera de Ingeniería de Mantenimiento Industrial. Riobamba, Ecuador. wilson.huilca@espoch.edu.ec

2 Escuela Superior Politécnica de Chimborazo, Facultad de Administración de Empresas, Carrera de Administración de Empresas. Riobamba, Ecuador. sharo@espoch.edu.ec
} 
del estudio es determinar si los tipos de filtros empleados en el sistema de alimentación de combustible afectan directamente en el rendimiento y eficiencia de un motor diésel, mediante el análisis matemático estadístico de los datos respecto a las fallas ocurridas causadas por las partículas contaminantes en los vehículos analizados que utilizan las 3 marcas de filtros recurrentes en la ciudad de Riobamba provincia de Chimborazo, declarando una hipótesis que afirma o niega, el cambio positivo de la eficiencia del motor, tomando en cuenta una muestra del parque automotor de la ciudad. Los resultados arrojados fueron que los tipos de filtros empleados no afectan en la eficiencia del motor, pudiendo ser otros componentes operacionales los que influyen en lo expuesto, por tal motivo se recalca que el presente estudio solo refleja una premisa probabilística mediante el resultado del análisis matemático estadístico, pudiendo servir en un futuro para una investigación exhaustiva de las condiciones y parámetros que influyen en la eficiencia de un motor.

Palabras claves: Eficiencia, Motor Diésel, Filtrado De Combustible, Análisis Matemático Estadístico, Impurezas

\section{Introducción}

Actualmente los vehículos con motor ya sea a gasolina o diésel son los predilectos dentro del parque automotor en Ecuador pese a que su eficiencia no sea tan elevada como la de los automóviles eléctricos. La eficiencia de filtrado es esencial a la hora de comprobar el rendimiento de un motor de combustible diésel, es por ello que el presente estudio pretende aceptar o rechazar la hipótesis que afirma que la eficiencia de un motor de combustible diésel depende del tipo de filtro utilizado, para ello se someterán los datos de fallos por filtro, de una muestra del parque automotor de la ciudad de Riobamba - Chimborazo a un análisis matemático estadístico, denotando que si dichos datos al ser sometidos a dicho análisis, cumplen con los valores esperados de las tablas estadísticas, se aceptará la hipótesis de lo contrario se rechazara la premisa.

Cabe recalcar que el presente estudio genera solamente un dato inicial, respecto a la eficiencia de un motor diésel, pero podrá servir como premisa de un estudio exhaustivo general en un análisis de eficiencia posterior

"Un motor diésel funciona mediante el encendido del combustible al ser inyectado muy pulverizado y con alta presión en una cámara de combustión que contiene aire a una temperatura superior a los motores de gasolina, esta es la llamada auto inflamación”. (Chiriboga, 2014)

"Los motores térmicos de combustión interna solamente son capaces de transformar entre el 35 y 50\%”, en energía y potencia (PALOMO \& PILATAX, 2019)

El proceso de combustión es el aspecto más importante de un motor de combustión interna, por lo que su modelado es de gran relevancia al simular el comportamiento global de un motor Diésel. "Los modelos de la combustión se suelen clasificar de acuerdo a la representación espacial del proceso". (Sánchez, 2013) 
Los vehículos a diésel a diferencia de los motores a gasolina son requeridos para realizar trabajos más pesados, tomando en cuenta que el valor de dicho combustible es menor que el de la gasolina.

"Un ciclo Diésel ideal es un modelo simplificado de lo que ocurre en un motor diésel. En un motor de esta clase, a diferencia de lo que ocurre en un motor de gasolina la combustión no se produce por la ignición de una chispa en el interior de la cámara". (UNIVERSIDAD DE SEVILLA, 2013)

En tal virtud se han desarrollado nuevas tecnologías, con la finalidad de mejorar el rendimiento y eficiencia de los motores diésel. La eficiencia del motor Diesel está directamente relacionado a factores tales como: el contexto operacional, la cantidad de aire que ingresa a la combustión, así como también el nivel de pureza del diésel.

Para garantizar un alto nivel de pureza requerido del diésel, los automóviles tienen incorporado un sistema de filtración pre-combustión, el mismo que se encuentra montado después de la bomba ubicada en el taque y antes del sistema de inyección del motor.

La característica específica del sistema inyector-bomba es que cada cilindro tiene asignada una bomba individual. Debido a ello la bomba y la tobera están combinadas en una pieza compacta que está instalada directamente en la cabeza del cilindro. "El sistema inyectorbomba es capaz de generar una presión de inyección de hasta 2200 bares". (Bosh, 2019)

"El sistema de inyección del motor es utilizado, obligatoriamente, en el ciclo del diésel desde siempre, puesto que el combustible tiene que ser inyectado dentro de la cámara en el momento de la combustión aunque no siempre la cámara está sobre la cabeza del pistón". (Nash, 2004)

El filtro de combustible (Diesel) desempeña funciones importantes para el suministro de combustible debido a la cantidad de contaminantes que tiene el combustible. "Los motores con sistemas de alimentación Diesel necesitan de una perfecta filtración del combustible debido a que la bomba de inyección y los inyectores son especialmente sensibles al agua". (Bosch, 2019)

"La reducción de las impurezas en forma de partículas es una de las funciones que debe cumplir el filtro de combustible". (Ferrer y Checa, 2019)

Así como también debe realizar una separación o extracción del agua que está incorporado en el diésel. "La separación del agua se efectúa en el elemento filtrante mediante un efecto repela demás de la extracción del agua cumple varias funciones adicionales. "Los módulos de filtración diésel desempeñan también otras funciones según los requerimientos específicos de cada aplicación: calentamiento de combustible, regulación de la presión, cebado, detección del nivel del agua, etc.” ("Filtros Diésel - Fram", 2019)

Uno de los agentes que influyen directamente con la durabilidad y vida útil del filtro es el combustible, ya que el diésel no tiene un proceso de refinación igual que la gasolina; con lo cual éste posee un número mayor de partículas y contaminantes. 
"El diésel que sale del crudo en los hornos tiene una cantidad de 9000 ppm de azufre, y al no ser un crudo tan pesado se lo pasa directamente a la estación de desulfuración de diésel HDS, posterior a esto se analiza su funcionamiento, eliminando el azufre del diésel. El diésel Premium para uso del parque automotor ecuatoriano cuenta con un bajo porcentaje de azufre $0.05 \%$, es decir 500ppm que están aceptadas por las normas anticontaminantes de la INEN, lo que conlleva a cumplir su calidad como combustible y su correcto desempeño en los motores diésel." (Ortiz, 2019)

El motor de combustión interna es una clase de máquina que transforma energía química, proveniente de un combustible, en energía mecánica. "El combustible arderá en el interior de una cámara especial de combustión”. (Menna, 2018)

La eficiencia de trabajo de un motor de combustión interna puede ser reflejado en sus gases de escape, ya que con la ayuda de una maquina especializada podemos analizar completamente los resultados obtenidos. La máquina es un analizador de emisiones de escape.

"Aparato capaz de medir, generalmente en porcentaje, determinados compuestos químicos contenidos en la masa de los productos, gaseosos o no, emitidos por el escape de un motor de combustión interna. El analizador puede ser de lectura directa o estar acoplado a un registrador, magnético o de otro tipo. La necesidad de analizar los gases de escape de los motores de combustión interna deriva del hecho que, sobre todo en los motores alternativos, la combustión de los hidrocarburos nunca es perfecta, razón por la cual los gases de escape no son solamente anhídrido carbónico $(\mathrm{CO} 2)$, agua $(\mathrm{H} 2 \mathrm{O})$, oxígeno $(\mathrm{O} 2)$ y nitrógeno $(\mathrm{N})$." ("Analizador de los gases De Escape - Definición - Significado", 2019)

Gracias a la ayuda de estos dispositivos podemos determinar cuál es el nivel exacto de emisiones que produce la máquina para poder compararlas con los niveles establecidos por las normas Euro. "Una norma europea sobre emisiones co2 es un conjunto de requisitos que regulan los límites aceptables para las emisiones de gases de combustión de los vehículos nuevos vendidos en los Estados Miembros de la UE. Las normas de emisión se definen en una serie de directivas de la UE con implantación progresiva que son cada vez más." ("Emisiones Co2: Euro 1, Euro 2, Euro 3, Euro 4", 2019). Estas normas Euro ha tenia ciertas variaciones con la finalidad de reajustar los estándares permisibles de emisiones. "Las etapas son normalmente denominadas Euro 1, Euro 2, Euro 3, Euro 4 y Euro 5 para vehículos ligeros. Las series correspondientes de las normas para vehículos pesados utilizan números romanos en vez de números arábigos (Euro I, Euro II, etc.)" ("Emisiones Co2, 2019).

Sin embargo, este tipo de nivel de emisiones puede ser controlado con la instalación de un filtro de partículas de escape, el cual se encarga de reducir dichos agentes. Sin embargo, desde el punto de vista interno del motor, éste sigue siendo ineficiente, y presenta un consumo sobre los límites establecidos. Por tal motivo se realiza el análisis del estado de los filtros internos de los motores como son: aire, aceite y combustible. Con la finalidad de obtener una mejora en el rendimiento y eficiencia. 
La corrosión en los motores de combustión interna sucede en parte por la formación de ácido sulfúrico que surge por la combinación del azufre que llevan los combustibles de menor calidad, con el agua y la humedad de la gasolina". (Bardhal 2018)

El agua repele el aceite lubricante que está encargado de proteger y reducir la fricción generada por el movimiento de las piezas metálicas. Además, la humedad puede adherirse a las piezas metálicas, oxidándolas.

"En los Diésel es muy importante su sustitución periódica ya que los sistemas de inyección directa a alta presión así como las bombas de inyección son elementos de altísima precisión mecánica y de un coste muy elevado de reparación o sustitución, por lo que asegurarse con cada cambio de filtro de combustible que al circuito de inyección no llegarán impurezas que puedan dañar el sistema, es vital para asegurar su longevidad y correcto funcionamiento". (Central Recambio Original, 2017)

\section{Filtro Bosch}

Características técnicas del filtro de diésel Bosch

- "Capacidad de retención de suciedad de 15 gramos*

- Medio filtrante de varias capas con una eficiencia del $99 \% * *$

- Caja del filtro fuerte de aluminio, plástico o de acero con superficie tratada, diseñada para soportar presión alta, ofreciendo así una vida útil más prolongada.

- Conexiones del filtro de combustible precisas y confiables para un filtrado de combustible del 100\%" ("Fuel Filters | Bosch Auto Parts", 2019)

Una de las formas de medir la eficiencia de un filtro de combustible (diésel) es teniendo en cuenta su índice beta el cual indica la capacidad de un filtro para retener partículas de ciertos tamaños que se encuentran en el combustible.

"La relación beta se establece contando el número de partículas de un tamaño específico que entran al filtro, y luego dividiéndolo por el número de partículas del mismo tamaño que salen del filtro." ("Baldwin Filters | Recomendaciones técnicas", 2019)

\section{Caterpillar}

"En los motores Caterpillar el Filtro de combustible primario retiene partículas de más de 10 micrones y evita que se tape el filtro secundario. El Filtro de combustible secundario retiene las partículas de 2 micrones y mayores con un $98 \%$ de eficiencia. De este modo, se reduce el desgaste de inyectores y bombas de combustible. Esto es esencial para los sistemas de alta presión de combustible y también extiende la vida útil de los sistemas de inyección más antiguos. El tener dos filtros es duplicar la vida útil del sistema de inyección. La filtración en serie se realiza con un filtro de 2 micrones en serie. En este caso el segundo filtro es como una "red de seguridad". Y así se duplica la vida útil de los inyectores". (Gruasytransportes.files.wordpress.com, 2019). 


\section{Nanonet}

"Nanonet presenta muchas capas de protección distintas para eliminar y retener partículas de hasta 4 micras (12 veces más pequeñas que la partícula de menor tamaño visible para el ojo humano) en una estructura de material compuesto." (Cumminsfiltration.com, 2019)

Posterior a la extracción de datos técnicos se procederá al cálculo de la muestra que se empleara para el análisis, además se considerará un análisis estadístico que nos permita establecer el número de fallas presentes a nivel provincial.

La finalidad de este procedimiento es lograr determinar cuántas partículas contaminantes son retenidas gracias al filtro de diésel de tal manera que se elimine todo rastro de los elementos perjudiciales dentro de la inyección y combustión de dicho combustible.

La comparación de los filtros de combustible se realizará entre las marcas Bosch, Caterpillar y Nanonet.

La siguiente tabla indica los valores mínimos y máximos que debe tener el diésel Premium en el país de acuerdo a la Ley de hidrocarburos.

Tabla 1. Características diésel Premium

\begin{tabular}{|c|c|c|c|c|}
\hline Requisitos & Unidad & Mínimo & Máximo & Método de ensayo \\
\hline Punto de inflación & ${ }^{\circ} \mathrm{C}$ & 40 & - & $\begin{array}{l}\text { NTE INEN } 1493 \\
\text { Procedimiento A }\end{array}$ \\
\hline$\varphi$ Contenido de agua y sedimento & $\%$ & - & 0,05 & NTE INEN 1494 \\
\hline $\begin{array}{l}\text { W Contenido de residuo carbonoso } \\
\text { sobre } 10 \% \text { del residuo de la destilación }\end{array}$ & $\%$ & - & 0,15 & NTE INEN 1491 \\
\hline W Contenido de cenizas & $\%$ & - & 0,01 & NTE INEN 1492 \\
\hline Temperatura de destilación del $90 \%{ }^{\circ} \mathrm{C}$ & ${ }^{\circ} \mathrm{C}$ & - & 288 & NTE INEN 926 \\
\hline Viscosidad cinemática a 37.8 & $\mathrm{cSt}$ & 1,3 & 3 & NTE INEN 810 \\
\hline W Contenido de azufre & $\%$ & - & 0,3 & ASTM 4294 \\
\hline $\begin{array}{l}\text { Corrosión a la lámina de cobre } \\
\text { Índice de cetano calculado }\end{array}$ & Clasificación & $\begin{array}{l}- \\
40\end{array}$ & $\begin{array}{c}\text { No.2 } \\
-\end{array}$ & $\begin{array}{l}\text { NTE INEN } 927 \\
\text { NTE INEN } 1495\end{array}$ \\
\hline
\end{tabular}

Fuente (Controlhidrocarburos.gob.ec, 2019) 
Tabla 2. Tabla de tipo de impurezas y daños en vehículos

\begin{tabular}{|c|c|c|c|}
\hline $\begin{array}{c}\text { Tipo de } \\
\text { impurezas }\end{array}$ & Impurezas & Origen & Daños \\
\hline \multirow{2}{*}{$\begin{array}{l}\text { Partículas } \\
\text { pesadas }\end{array}$} & $\begin{array}{l}\text { Materiales } \\
\text { minerales } \\
\text { (arena) }\end{array}$ & $\begin{array}{l}>\text { Polvo en la atmósfera } \\
>\text { Suciedad en el deposito } \\
>\text { Tubos de conducción }\end{array}$ & \multirow[b]{2}{*}{ Desgaste } \\
\hline & Óxido & $\begin{array}{l}\text { Oxidación del tanque de } \\
\text { combustible }\end{array}$ & \\
\hline $\begin{array}{l}\text { Partículas } \\
\text { livianas }\end{array}$ & $\begin{array}{l}\text { Abrasión } \\
\text { mecánica } \\
\text { Fibras } \\
\text { Partículas } \\
\text { plásticas }\end{array}$ & $\begin{array}{l}\text { Desgaste propio del sistema } \\
\text { Ambiente } \\
\text { Fragmentos de piezas del } \\
\text { sistema }\end{array}$ & $\begin{array}{l}\text { Obstrucción de los orificios } \\
\text { pequeños }\end{array}$ \\
\hline Micro partículas & & $\begin{array}{l}\text { Mangueras de suministro de } \\
\text { combustible }\end{array}$ & $\begin{array}{l}\text { Obstrucción del filtro } \\
\text { (ellos son depositados en } \\
\text { los componentes del } \\
\text { sistema de inyección) }\end{array}$ \\
\hline
\end{tabular}

Fuente: Controlhidrocarburos.gob.ec, 2019

Los costos de calidad inmersos son principalmente, los costos de prevención y los costos por materiales y servicios empleados, entre otros.

Tomando en cuenta que el valor del filtro no es elevado pero que puede traer consecuencias si no se realiza el mantenimiento de manera adecuada, los propietarios de automóviles prefieren realizar un mantenimiento completo en cuanto a filtros del automóvil, por lo tanto, se realiza cada dos cambios de aceite un cambio de filtros tanto de aire como de combustible. Este filtro de combustible puede tener un tiempo de vida variable ya que depende del tipo de combustible que se esté empleando.

Generalmente el cambio de filtro de combustible puede rondar entre las 3 a 5 veces por año dependiendo del recorrido que se vaya a ejecutar.

\section{Metodología}

Se debe tomar en consideración que los filtros a analizar deben cumplir con la retención del agua como de las partículas contaminantes tal que cumplan con las exigencias de la norma NTN INEN 1489:2012, norma que rige en el Ecuador.

Se debe destacar la importancia de generar un procedimiento analítico con el fin de establecer las comparaciones entre la eficiencia de filtrado.

Para el método de estudio, se requieren los siguientes procedimientos:

- Recolección de los datos de filtrado. 
- Ordenamiento de los datos.

- Cálculo de la muestra.

- Análisis mediante la herramienta estadística CHI cuadrado.

Cabe recalcar que el estudio está destinado a comparar la capacidad de filtrado de combustible por medio de la información obtenida por la base de datos del ente regulador de hidrocarburos.

El resultado esperado se basa en el principio estadístico de aprobar o rechazar la hipótesis, $\mathrm{H} 0$ no influye en el tipo de filtro y H1 si influye en el tipo de filtro, analizando todos los datos recogidos respecto a los fallos de las tres marcas de filtros utilizadas, Bosch, Caterpillar, Nanonet, dependerá del resultado de la aplicación de Chi cuadrado, para aceptar la hipótesis.

Cabe recalcar que el presente estudio toma una muestra de vehículos de la provincia de Chimborazo y solo permitirá obtener una premisa de información que servirá para otro estudio completo y exhaustivo respecto a los factores que implican en la eficiencia de un motor diésel

Por lo tanto después de obtener la muestra se observará al cabo de 3 meses, el comportamiento de fallos de 4 tipos de vehículos que trabajan con las diferentes micras de filtrado en sus componentes dele motor, este será posible realizarlo con la ayuda de una mecánica automotriz de la ciudad de Riobamba - Chimborazo que cuente con información diaria y un historial de fallos y reparaciones.

\section{Resultados}

Respecto al procesamiento de datos de los tipos de filtros.

Pariendo de los datos recolectados por el Instituto Nacional de Estadísticas y Censos se obtiene la población real de automóviles diésel que están registrados y matriculados en la provincia de Chimborazo.

Tabla 3. Vehículos registrados en Chimborazo

\begin{tabular}{|c|c|c|c|c|c|c|}
\hline \multirow{2}{*}{ PROVINCIA } & \multirow{2}{*}{ TIPO DE COMBUSTIBLE } & \multirow{2}{*}{ TOTAL } & \multicolumn{4}{|c|}{ USO } \\
\hline & & & Estado & Alquiler & Particular & Otros \\
\hline \multirow{5}{*}{ Chimborazo } & Diesel & 9.111 & 410 & 2.944 & 5.756 & 1 \\
\hline & Gasolina & 53.114 & 570 & 3.748 & 48.795 & 1 \\
\hline & Híbrido & 174 & - & - & 174 & - \\
\hline & Eléctrico & 1 & - & - & 1 & - \\
\hline & Gas Licuado de Petróleo & 4 & - & - & 4 & - \\
\hline
\end{tabular}

Fuente: INEC, 2019 


\section{Cálculo de la Muestra Analizar}

Para el cálculo de la muestra se toma en consideración los 9111 vehículos circulantes en la provincia.

El tamaño de la muestra por cálculos es:

$\mathrm{N}=9111$

$\mathrm{P}=5 \%$

$\mathrm{Z}=1,96$

$\mathrm{D}=0,5$

$\mathrm{Q}=1-\mathrm{P}=1-0,05=0,95$

$$
\begin{gathered}
n=\frac{N P Q Z^{2}}{N d^{2}+Z^{2} P Q} \\
n=\frac{(9.111)(0,05)(0,95)\left(1,96^{2}\right)}{(9.111)\left(0,05^{2}\right)+\left(1,96^{2}\right)(0,05)(0,95)}
\end{gathered}
$$

$n=79,63$

$\mathrm{n}=80$

Empleando los datos obtenidos en el cálculo de la muestra obtenemos que bastaría 80 automóviles o equipo caminero para determinar la viabilidad de emplear un filtro de mayor o menor capacidad de filtración.

\section{Datos de Filtros Empleados}

Respecto a los datos de los automotores mencionados anteriormente se tomó una muestra de varios ejemplares y se registrará la característica del sistema de filtración, así como también el tamaño de partículas que puede filtrar. Estos datos son de repuestos que se encuentran colocados al momento de dicho análisis, mas no se tomara datos de fábrica debido a que se a través de los años a estos automóviles se les ha hecho adecuaciones que evidencia su comportamiento en su desempeño.

Tabla 4. Filtros empleados por los automotores 
AUTOMOTOR

Tractor DRESSER

Retroexcavadora

CATERPILLAR

Camión mediano HYUNDAI HD45

Motoniveladora KOPMATSU

Camioneta VOLKWAGEN $4 \times 4$

Mini Van PEUGEOT
CARACTERISTICAS DE FILTRADO.

Filtro principal de 16 micrones, filtro auxiliar de 6 micrones, en serie con trampa de agua.

Filtro principal de 16 micrones, filtro auxiliar de 4 micrones, en serie con trampa de agua.

Filtro de 6 micrones más separador de agua

Filtro principal de 10 micrones, filtro auxiliar de 2 micrones, en serie con trampa de agua.

Filtro de 4 micrones

Filtro con tecnología de separación de agua de 4 micrones

Fuente: Autores

\section{Frecuencia de Cambio de Filtro Debido a Baja en Potencia}

Tabla 4. Tabla de frecuencia de cambio de filtro

\begin{tabular}{cc}
\hline AUTOMOTOR & FRECUENCIA DE CAMBIO \\
\hline $\begin{array}{c}\text { Tractor DRESSER } \\
\text { Retroexcavadora } \\
\text { CATERPILLAR }\end{array}$ & 200Horas de trabajo, 250 horas de trabajo \\
Camión mediano HYUNDAI & 220 horas de trabajo,250 horas de trabajo \\
HD45 & 10000 kilómetros de recorrido \\
Motoniveladora KOPMATSU & 250 horas de trabajo, 320 horas de trabajo \\
Camioneta VOLKWAGEN & 15000 kilómetros de recorrido \\
4X4 & 21000 kilómetros de recorrido \\
Mini Van PEUGEOT &
\end{tabular}

Fuente: Autores

\section{Determinación Estadística de Influencia de Filtros en Fallos de Automotores}

La tabla indica los valores correspondientes a las fallas por la capacidad de filtrado de combustible, haciendo referencia a la frecuencia con la que el filtro llega a colmatarse.

Tabla 5. Fallos por capacidad de filtrante (micras) 


\begin{tabular}{cccccc}
\hline & \multicolumn{3}{c}{ FALLOS POR CAPACIDAD FILTRANTE (micras) } & \multirow{2}{*}{ TOTAL } \\
\cline { 1 - 4 } TIPOS DE FILTROS & 2 & 4 & $6--8$ & oct-16 & \\
Bosch & 60 & 190 & 974 & 1900 & 3124 \\
Caterpillar & 63 & 176 & 945 & 1895 & 3079 \\
Nanonet & 59 & 183 & 936 & 1730 & 2908 \\
TOTAL & 182 & 549 & 2855 & 5525 & 911
\end{tabular}

Fuente: Autores

¿Influye el tipo de filtro con el número de fallos obtenidos?

Margen de error del 5\%

\section{Hipótesis}

H0 No influye el tipo de filtro

H1 Si influye el tipo de filtro con el que se encuentra trabajando el vehículo

Tabla 6. Frecuencia Teórica (micras)

\begin{tabular}{cccc}
\hline Para: & Frecuencia Teórica & Para: & Frecuencia Teórica \\
\hline 60 & 62,40 & 190 & 188,24 \\
63 & 61,51 & 176 & 185,53 \\
59 & 58,09 & 183 & 175,23 \\
& & & \\
Para: & Frecuencia Teórica & Para: & Frecuencia Teórica \\
974 & 978,93 & 1900 & 1894,42 \\
945 & 964,83 & 1895 & 1867,14 \\
936 & 911,24 & 1730 & 1763,44 \\
\hline
\end{tabular}

Fuente: Autores

\section{Grados de Libertad; 6}

Tabla 7. Análisis de la Hipótesis

\begin{tabular}{lcccccc}
\hline Frecuencia & 60 & 190 & 974 & 1900 & 63 & 176 \\
\hline $\begin{array}{c}\text { Frecuencia } \\
\text { Teórica }\end{array}$ & 62,4 & 188,24 & 978,93 & 1894,42 & 61,51 & 185,53 \\
$\quad$ Chi & 0,093 & 0,016 & 0,025 & 0,016 & 0,036 & 0,49 \\
$\begin{array}{c}\text { Cuadrado } \\
\text { Frecuencia }\end{array}$ & 945 & 1895 & 59 & 183 & 936 & 1730 \\
$\begin{array}{c}\text { Frecuencia } \\
\text { Teórica }\end{array}$ & 964,8 & 1867,1 & 58,09 & 175,23 & 911,24 & 1763,4 \\
$\quad$ Chi & 0,407 & 0,416 & 0,014 & 0,345 & 0,673 & 0,634 \\
Cuadrado & & & & & & \\
\hline
\end{tabular}

Fuente: Autores 
Tabla 8. Análisis de Resultados

\begin{tabular}{cc}
\hline CHI CUADRADO CALCULADO & 3,165 \\
\hline CHI CUADRADO DE TABLAS: & 12,592 \\
\hline
\end{tabular}

Fuente: Autor

A partir de una distribución de Chi cuadrado podemos determinar que se rechaza la Hipótesis Hl y se acepta HO. La cual manifiesta que no influye el tipo de filtro empleado en cada automóvil.

Con datos obtenidos de las características de los filtros, se puede establecer que en promedio debería emplearse un filtro de captación de partículas hasta de 4 micras, tomando en consideración que la tecnología nos permite un filtrado hasta de 2 micras.

\section{Discusión}

Como se observa al realizar la distribución $\mathrm{CHI}$ cuadrado se determina que el tipo de filtro empleado no es el principal causante al momento de analizar las fallas de un filtro.

Con la ayuda de una distribución estadística y considerando que se trata de un conjunto de características aleatorias-continuas presentes en un determinado intervalo de tiempo, la distribución Chi cuadrado se utiliza de tal manera que nos sirve como una herramienta para determinar estadísticamente: Sí el tipo filtro que se tiene instalado en un motor Diésel influye directamente con la cantidad de fallos que estos producen dentro de esta categoría de vehículos, indiferentemente del contexto operacional dónde estos se encuentren desarrollando podemos asimilar una tasa de fallos respectiva al tema de los filtros de combustible que se utilizan.

Cabe recalcar que un factor muy importante a considerar es la capacidad de filtrado de cada una de las marcas de filtros presentes en el estudio, aparte del análisis presentado un aspecto importante a tomar en cuenta sería el número de partículas contaminantes que cada filtro puede presentar ya que son las principales fuentes de desgaste dentro del sistema de alimentación de un motor de combustión interna.

\section{Conclusiones}

- El filtro de combustible es un elemento que cuenta con una vida útil limitada y que desempeña funciones importantes para obtener un buen rendimiento y eficiencia del motor diésel.

- Los filtros diésel pueden tener varias configuraciones, materiales, etc. que puede distinguir uno de otro.

- El tipo y marca de filtro de combustible no es necesario en el momento de que éste cumpla con su función, por lo que la gran parte de fallas se presentan a partir de la falta de mantenimiento.

- Para reducir la contaminación del diésel el cual afecte al rendimiento del motor se puede colocar sistemas de filtrados alternos el cual es incorporar dos filtros de distinto micraje en serie. 
- El cambio de filtro de combustible se debe realizar cada dos cambios del aceite del motor como máximo con el fin de garantizar un cumplimiento de funciones y así realizar la sustitución del mismo.

- Un filtro en mal estado puede generar una baja de potencia debido a la incapacidad de inyectar combustible en el volumen y proporción adecuada.

- Se recomienda que no se empleen filtros de micrajes pequeños sin contar con un filtro primario el cual recoja partículas más grandes, se puede utilizar una configuración de filtros de 7 micras con uno de 2 micras.

- Es recomendable que el dueño o propietario del automóvil ya sea pesado o liviano lleve un control en cuanto al mantenimiento de los filtros, componentes importantes del sistema.

\section{Referencias Bibliográficas}

Baldwin Filters (2019). Recomendaciones técnicas. Paldiski, Estonia. Recuperado de http://www.baldwinfilter.com/es/TechTips201210.html

BARDAHL. (2018). Bardahl. Toluca, México. Obtenido de https://www.bardahl.com.mx/riesgo-oxido-motor/

Bosch, R. (2019). Sistemas de inyección diésel por acumulador Como Rail. Madrid España. Recuperado https://books.google.com.ec/books?id=3Jl11hs3QiMC \&pg=PA24\&dq=filtros\%20de\%20co mbustible\&hl=es\&sa $=$ X\&ved=0ahUKEwjj9H54Z7iAhUE16wKHehKDGwQ6AEIKDAA\&fbclid=IwAR1sL3w69tn1F7NExc2Ux7D ykSaY1dsu-H1OUuT5-

2495WiEFIvzHF0n2G8\#v=onepage $\& \mathrm{q}=$ filtros $\% 20 \mathrm{de} \% 20$ combustible $\& \mathrm{f}=$ false

Central Recambio Original. (2017). Diferencias entre el mantenimiento de coche a diesel y gasolina. Barcelona España. Obtenido. de://www.recambiooriginal.com/blog/recambiosoriginales/mecanica/las-diferencias-mantenimiento-los-coches-diesel-gasolina/

Comparación de costos de mantenimiento del auto. (2019). Santa Cruz, Bolivia. Recuperado de https://www.widman.biz/mantenimiento/liviano.php

Chiriboga, E. (2014). Analisis de rendimiento y calidad del combustible a diesel. Argentina : Editex.

Cumminsfiltration.com. (2019). Filtration. Nashville Tenessee Recuperado de https://www.cumminsfiltration.com/sitemap

Emisiones Co2. (2019). Berlín Alemania. Recuperado de http://www.europasobreruedas.com/faq/emisiones-co2.html

Ferrer, J., y Checa, G. (2019). Mantenimiento mecánico preventivo del vehículo. Madrid España. Recuperado de https://books.google.com.ec/books?id=SwnFAwAAQBAJ\&pg=PA103\&dq=filtros\%20de \%20combustible\%20diesel\&hl=es\&sa=X\&ved=0ahUKEwiB8YyE4p7iAhVPcq0KHZDJB 
EQ6AEIODAD\&fbclid=IwAR1eibZR6rvTPAEmQdn9swMZqsYQ7OTXn4lxyFw0dzbKR cHK-KSRs571hzE\#v=onepage \&q=filtros\%20de\%20combustible\%20diesel $\& \mathrm{f}=$ false

Fuel Filters | Bosch Auto Parts. (2019). Tenessee USA. Recuperado de https://www.boschautoparts.com/es/auto/filters/fuel-filters

Gruasytransportes.files.wordpress.com. (2019). [online] Recuperado de https://gruasytransportes.files.wordpress.com/2016/01/caterpillar-contaminacion-decombustible-es.pdf

Menna. (2018). Motor de combustion interna. COMOFUNCIONA, 2 p.

MotorGiga (2019). Analizador de los gases de escape. Coruña España. Definición Significado. Recuperado de https://diccionario.motorgiga.com/diccionario/analizador-delos-gases-de-escape-definicion-significado/gmx-niv15-con187.htm

Nash, F. c. (2004). Fundamentos de mecanica automotriz. Mexico.

Ortiz, M. (2019). Estudio Comparativo del Uso del Diésel Entre Europa y Ecuador, Utilizado para Motores de Vehículos. Quito Ecuador. Recuperado de https://repositorio.uide.edu.ec/bitstream/37000/610/1/T-UIDE-0560.pdf

Palomo, K., y Pilatax, E. (2019). Diseño y construcción de un sistema de medición de consumo específico de combustible con la utilización de un software para la optimización del dinamómetro del laboratorio de motores diésel-gasolina. Quito Ecuador. Recuperado de https://repositorio.espe.edu.ec/bitstream/21000/8105/1/T-ESPEL-0980.pdf

Peugeot. (2002). Sistema HDI Anticontaminacion y pre-postcalentamiento diesel. España: CMA.

Sánchez, G. (2013). Modelado de la combustión en motores Diésel. Revista ION, p.42.

Universidad de Sevilla. (13 de Marzo de 2013). Wiki - Universidad de sevilla. Sevilla España. Obtenido de http://laplace.us.es/wiki/index.php/Ciclo_Diesel

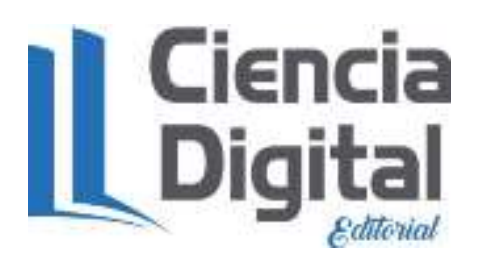

\section{PARA CITAR EL ARTÍCULO INDEXADO.}


Huilca Álvarez, A., \& Haro Acosta., S. (2019). Análisis de la eficiencia de un motor diésel, mediante el estudio estadístico de fallos de filtros de combustible. Ciencia Digital, 3(3), 431-445. https://doi.org/10.33262/cienciadigital.v3i3.660

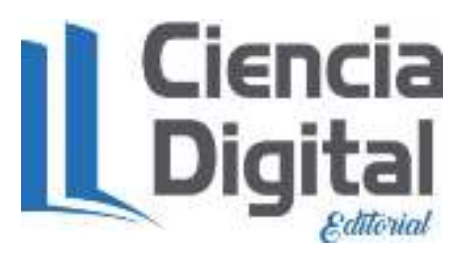

El artículo que se publica es de exclusiva responsabilidad de los autores y no necesariamente reflejan el pensamiento de la Revista Ciencia Digital.

El artículo queda en propiedad de la revista y, por tanto, su publicación parcial y/o total en otro medio tiene que ser autorizado por el director de la Revista Ciencia Digital.
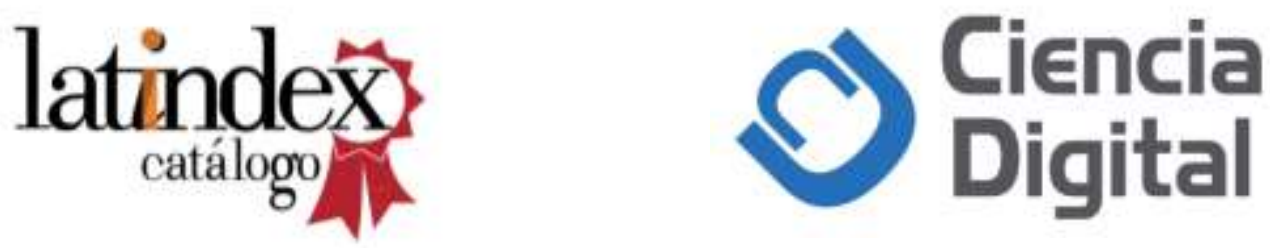